\title{
A PROBLEM OF HOOLEY IN DIOPHANTINE APPROXIMATION
}

\author{
by GLYN HARMAN
}

(Received 10 March, 1995)

1. Introduction. In [5] Professor Hooley announced without proof the following result which is a variant of well-known work by Heilbronn [4] and Danicic [3] (see [1]).

Let $k \geq 2$ be an integer, $b$ a fixed non-zero integer, and $\alpha$ an irrational real number. Then, for any $\epsilon>0$, there are infinitely many solutions to the inequality

$$
\left|\alpha n^{k}-m\right|<n^{-\rho(k)+\epsilon} \text { with }(b n, m)=1 \text {. }
$$

Here

$$
\rho(k)=\left\{\begin{array}{l}
\frac{2}{5} \text { if } \quad k=2 \\
\left(2^{k}-1\right)^{-1} \quad \text { if } k \geq 3 .
\end{array}\right.
$$

The difference between the above and previous work lies in the imposition of the condition $(b n, m)=1$ (in fact it is the condition $(n, m)=1$ which is of greater significance). This result was required to prove a theorem on approximating irrational numbers $\alpha$ by fractions of the form $v / \lambda$ where $f(v) \equiv 0(\bmod \lambda)$ for some given polynomial $f$ of odd degree exceeding 1 (Theorem 2 in [5], see our corollary below). The exponent $\rho(k)$ for $k \geq 3$ follows by combining Hooley's result in [6] with the usual Weyl inequality method, as is briefly sketched at the end of that paper. Here we shall prove the following stronger result. Our method also circumvents difficulties in the case $k=2$.

THEOREM. Let $k \geq 2$ be an integer, $b$ a fixed integer, and $\alpha$ an irrational real number. Then there are infinitely many solutions to (1) with

$$
\rho(k)=\left\{\begin{array}{l}
\frac{2}{5} \quad \text { if } \quad k=2 \\
\left(3.2^{k-2}-1\right)^{-1} \quad \text { if } \quad k \geq 3 .
\end{array}\right.
$$

Notes. For $k \geq 6$ better results follow from the work of Wooley [7]. It will be clear from our proof that the chief difficulty lies with large prime common factors of $m$ and $n$. This is the reason why our results fall short of the exponent $2^{1-k}$ given by the usual Heilbronn/Danicic approach. Using Wooley's work the integers $n$ are "smooth" (that is all their prime factors are less than $n \eta$ for some $\eta>0$ ) and so this difficulty does not arise. Recently significant progress has been achieved in the case $k=2$ [8], but it is not clear whether this new technique can be adapted to work in the present context.

COROLlaRY. Let $f(x)$ be a polynomial with integer coefficients having odd degree $k \geq 3$. Then, for any given real irrational $\alpha$, and any $\epsilon>0$, there are infinitely many moduli $\lambda$ for which there is a solution $v$ to $f(v) \equiv 0(\bmod \lambda)$ and

$$
\left\|\alpha-\frac{v}{\lambda}\right\|<\lambda^{-\tau(k)+\epsilon}
$$

Glasgow Math. J. 38 (1996) 299-308. 
with

$$
\tau(k)=\frac{1}{k}+\frac{\rho(k-1)}{k(k-1)}
$$

Here $\|x\|$ denotes the distance from $x$ to the nearest integer.

The corollary may be deduced from the theorem by the argument presented in [5] (for even $k$ a different argument is used which does not require results like our theorem).

We begin the proof by noting that for any function $f(x, y)$

$$
\sum_{(a, b)=1} f(a, b)=\sum_{a, b} f(a, b)-\sum_{p} \sum_{\substack{P(a, b) \geq p \\ \text { or }(a, b)=1}} f(p a, p b),
$$

where here and elsewhere in the paper $p$ and $r$ denote primes, and $P(m, n)$ denotes the smallest common prime factor of $m$ and $n$. If $(m, n)=1$ we put $P(m, n)=1$. The common factor $p$ in the second sum in (6) means that we are interested in bounding solutions to

$$
\left|\alpha n^{k} p^{k-1}-m\right|<p^{-1}(p n)^{-\rho(k)+e} \text {. }
$$

The problem with (7) is that although the right hand side is smaller (and so we expect fewer solutions than to (1)), the discrepancy between the exponents $k$ and $k-1$ raises problems. For $k \geq 3$ we shall find that an efficient strategy is to approximate $\alpha n^{k}$ by fractions with a suitably small denominator (see [2] where a similar approach is used). For $k=2$ we need to insist that $n$ has a rather special form at the outset.

As usual, we start with a convergent $a / q$ to $\alpha$ and consider

$$
\left|\frac{a n^{k}}{q}-m\right|<L^{-1}, \quad n \leq N, \quad(b n, m)=1, \quad L=2 N^{\rho(k)-\epsilon},
$$

where $N$ is chosen so that $q=\left(N^{k} L\right)^{1 / 2}$. It follows that if $n, m$ is a solution to (8) then

$$
\left|\alpha n^{k}-m\right|<\left(N^{k} L\right)^{-1} N^{k}+L^{-1}=2 L^{-1} \leq n^{-\rho(k)+\epsilon}
$$

as required.

2. Preliminary lemmas. We write $\mathcal{N}(N, \delta)$ for the number of solutions to

$$
\left\|\frac{b n^{k}}{s}\right\|<\delta, \quad n \leq N,
$$

and $\mathcal{N}(N, \delta, t)$ for the number of solutions to (9) with $(n, t)=1$.

Lемма 1. Let $s \geq 1$ and $b$ an integer coprime to $s$ be given. Let $\delta \in\left(0, \frac{1}{2}\right)$. Then, for $N \geq 1$ we have

$$
\mathcal{N}(N, \delta)=2 N \delta\left(1+O\left(N^{-\eta}\right)\right)+O\left(N^{1+2 \eta}\left(\frac{1}{N}+\frac{1}{s}+\frac{s \delta}{N^{k}}\right)^{\theta}\right)
$$

for any $\eta>0$, where $\theta=2^{1-k}$. Here the implied constant depends only on $\eta$ and $k$.

Proof. This result is implicit in the usual Heilbronn/Danicic treatment. See Chapter 3 of $[1]$. 
The bound (10) will sometimes have the great disadvantage that $N$ will be smaller than we would like (because we have extracted a large prime factor), but $\delta$ will be quite small. The $N^{1+2 \eta-\theta}$ term in the error thus becomes dominant. Professor Hooley has given a variant of the above result [6] where the main term is sacrificed, but the error term is improved (at least for small $\delta$ ). We state his result as follows. Without this result we would obtain $\rho(k)=\left(3.2^{k-2}\right)^{-1}$ for $k \geq 3$.

LEMMA 2. Under the hypotheses of Lemma 1 we have

$$
\mathcal{N}(N, \delta) \ll N\left(\frac{1}{N^{2}}+\delta+\frac{1}{s}+\frac{s \delta}{N^{k-\epsilon}}\right)^{\theta}
$$

We now state a variant of Lemma 1 which includes a coprimality condition. We write $\varphi(t)$ for Euler's totient function.

LeMma 3. Given the hypotheses of Lemma 1 together with a positive integer $t \leq N^{k}$. Then we have

$$
\mathcal{N}(N, \delta, t)=2 N \delta \frac{\varphi(t)}{t}\left(1+O\left(N^{-\eta}\right)\right)+O\left(N^{1+5 \eta}\left(\frac{1}{N}+\frac{1}{s}+\frac{s \delta}{N^{k}}\right)^{\theta}\right) .
$$

Proof. We make the dependence of $\mathcal{N}(N, \delta)$ on $b / s$ explicit, by rewriting the number of solutions to (9) as $\mathcal{M}(N, b / s)$. By Lemma 1 we then have

$$
\begin{aligned}
\mathcal{N}(N, \delta, t) & =\sum_{d \mid t} \mu(d) M\left(N / d, b d^{k} / s\right) \\
& =\sum_{d \mid t} \mu(d) \frac{2 N \delta}{d}\left(1+O\left(N^{-2 \eta}\right)\right)+O\left(\sum_{d \mid t} \frac{N^{1+4 \eta}}{d}\left(\frac{d}{N}+\frac{\left(d^{k}, s\right)}{s}+\frac{s \delta}{N^{k}}\right)^{\theta}\right) .
\end{aligned}
$$

The first term in (13) equals $2 N \delta \varphi(t)\left(1+O\left(N^{-\eta}\right)\right) / t$, while the second term leads to the error term in (12) since

$$
\sum_{d \mid t} \frac{\mu(d)}{d}=\frac{\varphi(t)}{t} \text { and } \sum_{d \mid t} 1 \ll t^{\eta / k} \leq N^{\eta}
$$

3. Proof of Theorem for $k \geqslant 3$. It is clear from (6) and (7) that problems will arise if there is some very good approximation, say $u / v$, to $a / q$, which gives rise to many approximations $m / n^{k}=u / v$. For large prime common factors this difficulty is dealt with by Lemma 5 below. We first prove the crucial step needed in Lemma 5 as a separate result. We put $K=2^{k-2}$ and write $a \sim A$ to mean $A \leq a<2 A$.

Lemma 4. Let $k \geq 3, \delta \in\left(0, \frac{1}{2}\right), J, H, N \geq 1$ be given, with

$$
J \geq \max \left(H^{K / 2}, N^{\epsilon} H^{K} \delta\right) \text { for some } \delta>0 .
$$

Then, for any real $\alpha$, we have that the number of solutions to

$$
\left|\alpha n^{k}-m\right|<\delta, \quad n \leq N, \quad P(m, n) \geq J
$$

is

$$
\ll \frac{N \log N}{H}+\sum_{R=2^{i}, j=0,1, \ldots} R \mathcal{N}\left(N / R, \delta / R^{k}, Q(R)\right)
$$


Here

$$
Q(R)=\prod_{p<R} p .
$$

Proof. Let $r=P(m, n)$. We consider the contribution to the number of solutions of (15) with $r \sim R=2^{j}$. We thus wish to bound the number of solutions to

$$
\left|\alpha v^{k} r^{k-1}-m\right|<\delta / R \text {, with } v \leq N / R \text {. }
$$

Here and in the following we suppress the condition $(v, m)$ coprime to $Q(R)$ for the sake of clarity. Let $T=(2 R)^{k} \delta^{-1} H^{-K} N^{-\epsilon}\left(\geq 2^{k} R^{k-1}\right.$ by (14)). By Dirichlet's theorem in Diophantine approximation, for each $v$ there are integers $b, s$ with $(b, s)=1$ and

$$
\left|\alpha v^{k}-\frac{b}{s}\right|<\frac{1}{s T}, \quad 1 \leq s \leq T .
$$

We deal first with the case $2 s \geq H^{K}$. We have

$$
\left\|\alpha v^{k} r^{k-1}\right\|=\left\|\frac{b r^{k-1}}{s}\right\|+O\left(\frac{R^{k-1}}{s T}\right)=\left\|\frac{b r^{k-1}}{s}\right\|+O\left(N^{\epsilon} \delta / R\right) .
$$

Hence the number of solutions to (17) for each $v$ with $2 s \geq H^{K}$ can be estimated by Lemma 2 as

$$
\begin{aligned}
& \ll R\left(\frac{1}{R^{2}}+\frac{N^{\epsilon} \delta}{R}+\frac{2}{H^{K}}+\frac{s \delta N^{\epsilon}}{R^{k-1}}\right)^{1 / K} \\
& \ll \frac{R}{H} .
\end{aligned}
$$

Multiplying this by $N / R$ and summing over $\ll \log N$ values for $R$ gives the first term in (16).

The case $2 s \ll H^{K}$ needs to be split into three subcases as follows.

(i) $s \nmid r^{k-1}$. We note that

$$
\left\|\alpha v^{k} r^{k-1}\right\| \geq\left\|\frac{b r^{k-1}}{s}\right\|-\frac{(2 R)^{k-1}}{T s} \geq \frac{1}{s}\left(1-\frac{(2 R)^{k-1}}{T}\right) \geq \frac{1}{2 s} \geq H^{-K}>\frac{\delta}{R} .
$$

Here we have used $T \geq 2^{k} R^{k-1}$ and $R H^{-K} \geq J H^{-K} \geq \delta N^{\epsilon}$ by (14). It follows from (18) that there can be no solutions to (17) arising from this case.

(ii) $s \neq 1, s \mid r^{k-1}$. Since $r$ is a prime, there is at most one solution for each given $s$. Hence there can be at most $N / R$ solutions to (17) arising from this case. Summing over $R$ then gives $\ll N / J \leq N / H$ solutions as required.

(iii) $s=1$. Since $(2 R)^{k-1} T^{-1} \leq \frac{1}{2}$, if

$$
\left|\alpha v^{k}-b\right|<T^{-1} \text { and }\left\|\alpha v^{k} r^{k-1}\right\|<\delta / R \text {, }
$$

we must have

$$
\left|\alpha v^{k}-b\right|<\delta R^{-k} \text {. }
$$

For each solution to (19) trivially there will be at most $R$ solutions in $r$ to (17). This leads 
to the sum in (16) and completes the proof. Note that $m=b r^{k-1}$ so $(v, h)$ is coprime to $Q(R)$ as claimed.

Lemma 5. Let $k \geq 1, a, q, L, N$ be as given in Section 1. Then either

(i) there is a solution to

$$
\left|\frac{a n^{k}}{q}-m\right|<L^{-K}, \quad(m, n)=1, \quad n \leq N L^{1-K} ;
$$

(ii) the number of solutions to

$$
\left|\frac{a n^{k}}{q}-m\right|<\frac{1}{L}, \quad(n, q)=1, \quad n \leq N, \quad P(m, n)>L^{K-1} N^{\epsilon}
$$

is

$$
\ll N^{1-\eta} L^{-1} \text { where } \eta=2^{-k-2} \epsilon .
$$

Proof. Suppose (i) does not hold. We then apply Lemma 4 with $J=L^{K-1} N^{\epsilon}, N_{1}=N$, $\delta_{1}=1 / L, H=L N^{2 \eta}$. By (16) we get a bound $\ll N^{1-\eta} L^{-1}$ plus a sum involving the solutions to

$$
\left|\frac{a n^{k}}{q}-m\right|<\frac{1}{L R^{k}}, \quad P(m, n)>J, \quad n \leq N / R, \quad(n, q)=1 .
$$

Here we have noted that since (i) fails the condition $(Q(J), m, n)=1$ implies $P(m, n)>J$, and the addition of $(n, q)=1$ to Lemma 4 carries through the proof. We also remark that since $(n, q)=1$ we must have

$$
\left\|\frac{a n^{k}}{q}\right\| \geq \frac{1}{q}
$$

and so we need only consider values of $R$ with $L R^{k}<q$. We now apply Lemma 4 again with $\delta_{2}=L^{-1} R^{-k}, N_{2}=N / R$. This gives $\ll N^{1-2 \eta} /(H R)$ solutions plus those coming from a further inequality to which Lemma 4 can be applied again with $\delta_{3}=\left(L R^{k} U^{k}\right)^{-1}$, $N_{3}=N /(R U)$, where $U \geq R, L(U R)^{k} \leq q$. At this point the argument stops because we are left to consider solutions to

$$
\left|\frac{a v^{k}}{q}-b\right|<L^{-1}(R U V)^{-k}, \quad v \leq N /(R U V), \quad(v, q)=1,
$$

where $V \geq U \geq R$. Since

$$
\frac{J^{3 k} L}{q} \geq J^{3 k} N^{-k / 2} \geq N^{y}
$$

with

$$
y=\frac{3 k\left(2^{k-2}-1\right)}{3 \cdot 2^{k-2}-1}-\frac{k}{2}>0
$$

the only solutions to $(24)$ have $v^{k} \equiv 0(\bmod q)$, but this is ruled out by the condition $(v, q)=1$. 
The total number of solutions to (21) is thus

$$
\frac{N \log N}{H}+\sum_{R} R\left(\frac{N}{H R} \log N+\sum_{U} U \frac{N}{H R U} \log n\right) \ll \frac{N^{1-\eta}}{L}
$$

as required.

Proof of Theorem for $k \geq 3$. To simplify the proof we suppose that $b=1$ in the following. If there are infinitely many solutions to (20) for infinitely many $a / q$ the result follows. Otherwise suppose that (20) is false for $a / q$. Let $\delta=L^{-1}$. The number of solutions to

$$
\left|\frac{a n^{k}}{q}-m\right|<\delta, \quad(m, n)=1, \quad n \leq N, \quad(n, q)=1
$$

is (using the notation of $\S 2$ with $b / s=a / q$ )

$$
\mathcal{N}(N, \delta, q)-\sum_{\substack{p \geq 2 \\(p, q)=1}} \mathscr{A}(p)
$$

where $\mathscr{A}(p)$ denotes the number of solutions to (25) with $P(m, n)=p$. The first term in (26) is

$$
2 N \delta \frac{\varphi(q)}{q}\left(1+O\left(N^{-\eta}\right)\right)
$$

with $\eta=2^{-k-2} \epsilon$ by Lemma 3. Now take $b / s=a p^{k-1} / q$ in the definition of $\mathcal{N}(N, \delta, t)$. Then that part of the sum in (26) with $p \leq L^{K-1} N^{\epsilon}$ can be bounded above by

$$
\begin{aligned}
\sum_{\substack{2 \leq p \leq L^{K-1} N^{*} \\
(p, q)=1}} \mathcal{N}(N / p, \delta / p, q)=\sum_{\substack{2 \leq p \leq L^{K-1} N^{*} \\
(p, q)=1}} 2 N \delta \frac{\varphi(q)}{q p^{2}}\left(1+O\left(N^{-\eta}\right)\right) \\
+O\left(\sum_{\substack{2 \leq p \leq L^{K-1} N^{\cdot} \\
(p, q)=1}}(N / p)^{1+5 \eta}\left(\frac{p}{N}+\frac{1}{q}+\frac{q p^{k-1} \delta}{N^{k}}\right)^{\theta}\right)
\end{aligned}
$$

using Lemma 3. The error term is $O\left(\delta N^{-2 \eta}\right)$ since

$$
\frac{L^{K-1} N^{\epsilon}}{N} \leq \frac{N^{-\epsilon}}{L^{2 K}}, \text { and } \frac{q\left(L^{K-1} N^{\epsilon}\right)^{k-1}}{N^{k}} \leq \frac{N^{-\epsilon}}{L^{2 K}}
$$

It is the first inequality in (28) which is more significant and leads to the quoted value for $\rho(k)$. For the part of the sum in (26) with $p>L^{K-1} N^{\epsilon}$ we use Lemma 5. We thus obtain that the number of solutions to $(25)$ is

$$
\geq 2 N \delta \frac{\varphi(q)}{q}\left(1-\sum_{p \geq 2} \frac{1}{p^{2}}+O\left(\frac{q N^{-\eta}}{\varphi(q)}\right)\right)>0
$$

for all large $N$. This completes the proof for $k \geq 3$. 
4. The case $k=2$. We now only count solutions $n$ which have a prime factor of size around $N^{1 / 5}$. This enables us to use the following result.

Lemma 6. Let $a, q, L, N$ be as given in Section 1 with $L>32$. Suppose that $2 \leq D, P$, with $D P<N, P>N^{1 / 6}$. Then the number of solutions to

$$
\left\|\frac{a r n^{2} p^{2}}{q}\right\|<\frac{1}{L D}, \quad(n p, q)=1, \quad p \sim P, \quad r \sim D, \quad n \leq N /(D P),
$$

is

$$
\ll \frac{N}{L}\left(\frac{P}{D}+\frac{q N^{\eta}}{N P}+\frac{q N^{\eta}}{N D}+\frac{L}{D P}\right)
$$

for any $\eta>0$.

Proof. Since (30) exceeds $N /(D P)>1$, we may assume that at least one solution to (29) exists for each $n$. It follows that for some $n, p, r$ restricted as in (29) we have

$$
\left|\frac{n^{2} a}{q}-\frac{b}{r p^{2}}\right|<\frac{1}{L D^{2} P^{2}}
$$

Now we rewrite $b / r p^{2}$ as $c / d$ with $(c, d)=1$ and consider the possible values for $d$ (since $p, r$ are primes there are only 6 possible values for $d$ ).

(i) $d=p^{2} r$. We count the number of solutions to

$$
\left\|\frac{n^{2} a f}{q}\right\|<\frac{1}{L D} \text { with } f<8 P^{2} D .
$$

We have

$$
\left\|\frac{n^{2} a f}{q}\right\|=\left\|\frac{c f}{r p^{2}}\right\|+O\left(\frac{1}{L D}\right) .
$$

It follows that (31) will only have solutions if

$$
c f \equiv h\left(\bmod p^{2} r\right) \quad \text { with } \quad|h| \ll 1 / L D .
$$

There are therefore $\ll 1+\left(P^{2} D\right) / L D=1+P^{2} / L$ possible solutions for each $n$. Summing over the $N / P D$ possible values for $n$ then gives a bound

$$
\ll \frac{N}{L D}+\frac{N P}{L D}
$$

which is suitable in view of (30).

(ii) $d=p r$. Suppose there is another solution $u=v w$ with $t / u=b / r p^{2}$. Then

$$
\left|\frac{t}{u}-\frac{c}{d}\right| \leq\left|\frac{n^{2} a}{q}-\frac{c}{d}\right|+\left|\frac{n^{2} a}{q}-\frac{u}{v}\right|<2\left(L D^{2} P^{2}\right)^{-1} \leq(u d)^{-1} .
$$

Hence $t / u=c / d$, which means $v=r, w=p$. There are thus at most two solutions for each $n$ in this case, which leads to $\ll N / P D$ solutions altogether. 
(iii) $d=p$ or $p^{2}$. In this case we have

$$
\left\|\frac{(p n)^{2} a}{q}\right\|<\frac{1}{L D^{2}}
$$

We can therefore deal with this case by multiplying by $D$ (a very generous upper bound for the number of primes $r \sim D$ !) the number of solutions to

$$
\left\|\frac{m^{2} a}{q}\right\|<\frac{1}{L D^{2}}, \quad m \leq 2 N / D, \quad(m, q)=1 .
$$

(The number of solutions to $p n=m$ is no more than 5 since $p>N^{1 / 6}$ ). We bound this number by considering the congruence

$$
m^{2} a \equiv h(\bmod q), \quad 1 \leq|h| \leq q / L D^{2} .
$$

For each fixed $h$ there are $\ll N^{\eta}$ solutions to $m^{2} a \equiv h(\bmod q)$ and so we finally obtain a bound

$$
\ll \frac{N^{\eta} q}{L D^{2}} D=\frac{N^{1+\eta}}{L}\left(\frac{q}{N D}\right)
$$

which gives the third term in (30).

(iv) $d=r$. We now must have

$$
\left\|\frac{\operatorname{ran}^{2}}{q}\right\|<\left(L D P^{2}\right)^{-1}
$$

For each fixed $r$ we may argue as in (iii) to show that the number of solutions to (32) is $\ll N^{\eta} q\left(L D P^{2}\right)^{-1}$. Multiplying by PD then gives the second term in (30).

(v) $d=1$. We now count $P D$ times the number of solutions to

$$
\left\|\frac{n^{2} a}{q}\right\|<\left(L D^{2} P^{2}\right)^{-1}, \quad(n, q)=1 .
$$

We may argue as in case (iii) again eventually arriving at a bound $\ll N^{\eta} q(L D P)^{-1}$, which is smaller than either of the terms from (iii) and (iv). This completes the proof.

Proof of Theorem for $k=2$. Write $P=L^{1 / 2} N^{4 \eta}, R=L^{1 / 2} N^{6 \eta}$ with $\eta=\epsilon / 100$. We count solutions to

$$
\left|\frac{a n^{2} p^{2}}{q}\right|<\frac{1}{L}, \quad n \leq N / p, \quad p \sim P, \quad(n p, m q)=1, \quad(n, p)=1 .
$$

As before we write $\delta=1 / L$. In the following $p$ is always to be taken as coprime to $q$. The number of solutions to (33) is $S_{1}-S_{2}-S_{3}$ where $S_{1}$ counts solutions to

$$
\left\|\frac{a n^{2} p^{2}}{q}\right\|<\delta
$$

$S_{2}$ counts solutions to

$$
\left\|\frac{a n^{2} p}{q}\right\|<\delta / p
$$


and $S_{3}$ counts solutions to

Now let

$$
\left|\frac{p^{2} n^{2} a r}{q}-\frac{m}{r}\right|<\delta / r, \quad r \neq p, \quad P(m, n)>r
$$

Then, by Lemma 3 ,

$$
\pi=\sum_{p \rightarrow P} \frac{1}{p} \approx \frac{1}{\log P}
$$

$$
\begin{aligned}
S_{1} & =\sum_{p-P} \frac{2 N \delta}{p} \frac{\varphi(p q)}{p q}\left(1+O\left(N^{-\eta}\right)\right)+O\left(\sum_{p \sim P} \frac{N^{1+5 \eta}}{P}\left(\frac{P}{N}+\frac{1}{q}+\frac{P^{2} \delta q}{N^{2}}\right)^{1 / 2}\right) \\
& =\frac{2 N \varphi(q) \delta}{q}\left(1+O\left(N^{-\eta}\right)\right) \pi .
\end{aligned}
$$

Here we have observed that $\varphi(p q) / p q=(1+O(1 / p)) \varphi(q) / q$, and $L=N^{2 / 5-\epsilon}$ gives

$$
P<\delta^{2} N^{1-\epsilon} \text { and } P^{2} q<\delta N^{2-\epsilon} .
$$

Applying Lemma 3 to $S_{2}$ gives the formula

$$
S_{2}=\sum_{p \sim P} \frac{2 N \delta}{p^{2}} \frac{\varphi(p q)}{p q}\left(1+O\left(N^{-\eta}\right)\right)+O\left(\sum_{p \sim P} \frac{N^{1+5 \eta}}{P}\left(\frac{P}{N}+\frac{1}{q}+\frac{P \delta q}{N^{2}}\right)^{1 / 2}\right) \ll \frac{N^{1-\eta} \varphi(q) \delta \pi}{q}
$$

arguing as for $S_{1}$.

We split $S_{3}$ into 3 subsums $S_{4}, S_{5}, S_{6}$ with $r \leq N^{\eta}, N^{\eta}<r \leq R, r>R$ respectively. By the argument used for $S_{1}$ we have

$$
S_{4} \leq T=\frac{2 N \varphi(q) \pi \delta}{q} \sum_{2 \leq r \leq N^{n}} \frac{1}{r^{2}} \quad\left(1+O\left(N^{-\eta}\right)\right)
$$

Here $T$ is the number of solutions $n, p, r$ such that $r \neq p$ and

$$
\left\|\frac{p^{2} n^{2} r a}{q}\right\|<\frac{\delta}{r}
$$

For $S_{5}$ we count solutions to

$$
\left\|\frac{m^{2} a}{q}\right\|<\frac{\delta}{r}, \quad m \leq \frac{N}{r}, \quad(m, q)=1 .
$$

An application of Lemma 3 yields an upper bound

since by our choice of $R$ we have $R<\delta^{2} N^{1-\epsilon}$ and $R q<N^{2-\epsilon}$.

$$
\ll \frac{N^{1-\eta} \varphi(q) \delta}{q}
$$

For $S_{6}$ we apply Lemma 6 to obtain

$$
S_{6} \ll \sum_{\substack{D=2^{i} R \\ j=0,1, \ldots}} N \delta\left(\frac{P}{D}+\frac{q N^{\eta}}{N P}+\frac{q N^{\eta}}{N D}+\frac{1}{D P \delta}\right) \ll \delta N^{1-2 \eta} \ll \frac{N^{1-\eta} \varphi(q) \delta}{q} .
$$

Hence the number of solutions to $(33)$ is

$$
\geq \frac{2 N \delta \varphi(q) \pi}{q}\left(1-\sum_{r \geq 2} \frac{1}{r^{2}}+O\left(N^{-\eta}\right)\right)
$$

which completes the proof. 
ACKnowledgement. The author wishes to thank Professor Hooley for drawing his attention to this problem and for the interesting discussions that followed.

\section{REFERENCES}

1. R. C. Baker, Diophantine inequalities, London Math. Soc. Monographs N.S.1 (Oxford Science Publications, 1986).

2. R. C. Baker and G. Harman, On the distribution of $\alpha p^{k}$ modulo one, Mathematika 38 (1991), 170-184.

3. I. Dancic, An extension of a theorem of Heilbronn, Mathematika 5 (1958), 30-37.

4. H. A. Heilbronn, On the distribution of the sequence $\theta n^{2}(\bmod 1)$, Quart. J. Math. Oxford Ser. 2, 19 (1948), 249-256.

5. C. Hooley, On the location of the roots of polynomial congruences, Glasgow Math. J. 32 (1990), 309-316.

6. C. Hooley, On an elementary inequality in the theory of Diophantine approximation, in Analytic Number Theory, Proceedings of a conference in honour of Heini Hallerstam (Birkhauser, 1996), 471-486.

7. T. D. Wooley, The application of a new mean value theorem to the fractional parts of polynomials, Acta Arith. 65 (1993), 163-179.

8. A. Zaharescu, Small values of $\alpha n^{2}(\bmod 1)$, Invent. Math. 121 (1995), 379-388.

School of Mathematics

23 SENGHENNYDD ROAD

P.O. Box 926

CARDIFF CF2 4YH

WALES

e-mail: Harman@cf.ac.nk 